\title{
Transforaminal Endoscopic Lumbar Discectomy with Foraminoplasty for Down-migrated Disc Herniation: A Single-center Observational Study
}

\author{
Ching-Ting Tai ${ }^{1}$, Kuo-Tai Chen ${ }^{2}$, Shang-Wun Jhang ${ }^{3}$, Guan-Chyuan Wang ${ }^{4}$, Kai-Sheng Chang ${ }^{3}$, Li-wei Sun ${ }^{3}$, \\ Chien-Min Chen ${ }^{3,5,6}$ \\ ${ }^{1}$ Department of Family Medicine, Lukang Christian Hospital, Changhua, Taiwan \\ ${ }^{2}$ Department of Neurosurgery, Chang Gung Memorial Hospital Chiayi Branch, Chiayi, Taiwan \\ ${ }^{3}$ Division of Neurosurgery, Department of Surgery, Changhua Christian Hospital, Changhua, Taiwan \\ ${ }^{4}$ Division of Neurosurgery, Department of Surgery, Mennonite Christian Hospital, Hualien, Taiwan \\ ${ }^{5}$ School of Medicine, Kaohsiung Medical University, Kaohsiung, Taiwan \\ ${ }^{6}$ College of Nursing and Health Sciences, Dayeh University, Changhua, Taiwan
}

Received: November 27, 2021

Accepted: January 2, 2022

Corresponding Author:

Chien-Min Chen, MD, PhD

Division of Neurosurgery,

Department of Surgery, Changhua

Christian Hospital, No. 135 Nanxiao

St., Changhua City, Changhua

County 500, Taiwan

Tel: +886-4-7238595

Fax: +886-4-7232942

E-mail:96015@cch.org.tw
Objective: Full-endoscopic lumbar discectomy has evolved to be an alternative for the treatment of lumbar disc herniation. Regarding the techniques, the transforaminal approach remains the primary access. The indications of transforaminal endoscopic lumbar discectomy (TELD) have expanded following the evolution of the techniques, especially TELD with foraminoplasty. This study is to evaluate the efficacy of the TELD with foraminoplasty for downward migrated lumbar disc herniation.

Methods: The authors conducted a retrospective study with prospectively collected data in a single center. The study enrolled patients with downward migrated lumbar disc herniation undergoing TELD with foraminoplasty from May 2009 to June 2018. All procedures were performed under local anesthesia. Patients' demographics, clinical outcomes, and satisfaction with surgery were recorded.

Results: There were 126 patients included in the current study. The mean age was $50.7 \pm 17.4$ years old. The leg pain and functional outcome scores significantly improved after the operation. There was no neurological deficit or iatrogenic instability requiring fusion surgery. The operation time was within 2 hours in most cases (92\%). Thirteen patients reported minor complications, but symptoms were self-limited or responded to conservative treatment. The operation satisfied $94.4 \%$ of patients. Seven cases underwent revision surgery within six months due to recurrence. Conclusion: TELD with foraminoplasty under local anesthesia can be an alternative for downward migrated lumbar disc herniation. Nerve root irritation can be detected without intraoperative neurophysiological monitoring when the patient is awake during the procedures. The clinical outcomes were favorable and the risk of complication was low with the current technique.

Key Words: Endoscopes, Discectomy, Percutaneous, Disc herniation, Slipped disc, Enhanced recovery after surgery 


\section{INTRODUCTION}

Transforaminal endoscopic lumbar discectomy (TELD) has been an alternative for minimally invasive surgery of the soft lumbar disc herniation [1-6]. TELD is usually conducted under local anesthesia with or without conscious sedation to decrease exiting nerve root injury during the procedures. Comparing with open discectomy, TELD is superior in minimizing injury to collateral soft tissue [7], which benefits postoperative pain, shorter hospital stay, and early return to daily life or work [8-11].

With the evolution of surgical instruments and techniques, the indications of TELD have been expanded to treat migrated disc or combined lumbar foraminal stenosis $[12,13]$. The core procedure to expand the application of TELD is foraminoplasty, which can widen the narrow foramen to allow the working cannula to pass through easily $[14,15]$. Foraminoplasty can increase the safe working zone by resecting the ventrolateral aspect of the superior articular process (SAP) to avoid injury to the exiting nerve root [16]. However, patients might experience severe pain and cannot cooperate with the surgery during complicated procedures if the local anesthesia is inadequate.

The previous studies mainly focused on different modifications of transforaminal endoscopic techniques. Some authors have described the endoscopic foraminoplasty technique by using a drill or reamer in performing TELD $[14,15,17]$. Nevertheless, there is a lack of study describing the stepwise anesthetic techniques. Adequate local anesthesia is the first step of the operation and is essential to patients' satisfaction. The standard anesthetic technique of TELD is epidural block before puncturing through annulus and discography. The target and procedures of TELD with foraminoplasty are different from the standard TELD. Therefore, the authors will report their modified technique and patient-reported outcomes in the study.

\section{MATERIALS AND METHODS}

The Institutional Review Board approved the study (IRB No. 190905), and all patients had informed consent. The retrospective study enrolled patients with downward migrated lumbar disc herniation undergoing TELD with foraminoplasty in a single institute. Patients having a previous lumbar operation or spondylolisthesis were excluded. The demographics and patient-reported outcomes were recorded prospectively. The operations were done by a single surgeon (corresponding author). The surgical technique was modified from TESSYS with a single endoscopic spine system (VANTAGE BIOTECH CO., LTD., Taoyuan, Taiwan). The clinical outcomes were assessed with the visual analogue scale (VAS) and Oswestry Disability Index (ODI). The patients' satisfaction with the surgery was evaluated on postoperative day 1 . The patient satisfaction was categorized as "excellent", "good", "fair", and "poor".

Statistical analysis was conducted using IBM SPSS Statistics (Version 22.0). Continuous variables were expressed as the mean \pm standard deviation. A paired t-test was used to compare preoperative and postoperative results. A p-value $<0.05$ was considered statistically significant.

\section{TELD with Foraminoplasty Protocol}

\section{1) Preparation}

A standardized patient education sheet and video clips were provided to patients in the neurosurgical clinic before surgery. Information about the surgery, expectations, and support services was available on the education sheet. The education was reinforced at the preoperative service.

The patient is in the prone position with hips and knees flexion on the Jackson table. C-arm fluoroscopy was used to verify the herniated segment. The entry point on the skin was determined according to the TESSYS technique proposed by Schubert and Hoogland [17].

\section{2) Stepwise Anesthesia and Docking the Working Cannula}

After planning the entry point and trajectory, the patient is given local anesthesia with 3 to $5 \mathrm{~mL} 1 \%$ lidocaine at the subcutaneous and fascia layer. Then, a stab incision by $8 \mathrm{~mm}$ is made through the skin and fascia with a blade. The cannulated needle is inserted from the entry point. During inserting the needle to the lateral SAP, about 5 to $10 \mathrm{~mL} 1 \%$ lidocaine was infiltrated in the muscle layer (Figure 1). The target of the needle placement is at the junction of the SAP and pedicle, which are away from the exiting root and identified by fluoroscopic guidance easily (Figure 2). Thus, epidurogram and discogram can be omitted. Then, the needle was withdrawn slightly and then inserted at a higher inclination angle through the ventral SAP (Figure 3). After confirming the needle position by fluoroscopy, about 5 to $10 \mathrm{~mL} 0.5 \%$ lidocaine is given to infiltrate and block the ventral facet. Although the diluted lidocaine may fail to infiltrate the epidural space adequately, the pain from irritation to the annulus is minimal with the current technique. Then, a guidewire is inserted through the cannulated needle and followed by sequential dilators to create the track for a working cannula. Patients were awake during the whole procedure and could report their discomfort immediately. The working channel is placed through the dilator and docked on the ventrolater- 

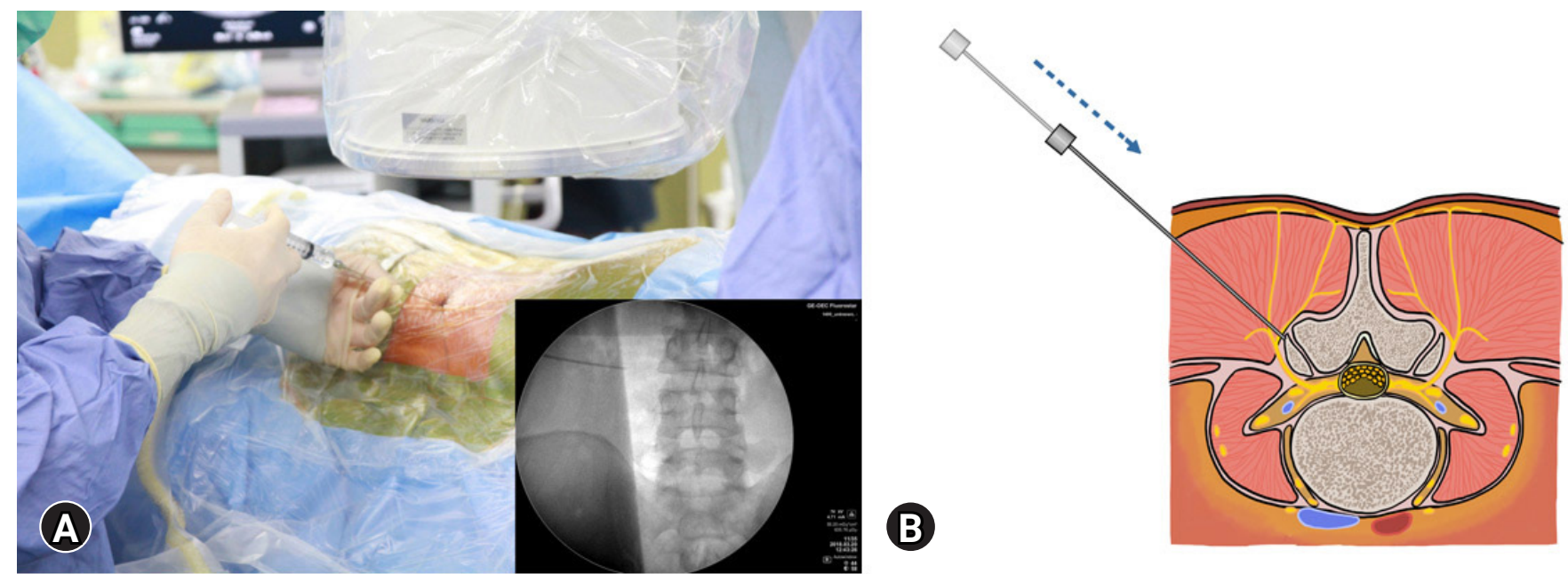

Figure 1. (A) The cannulated needle was inserted from skin through muscle to lateral SAP under C-arm fluoroscopy guidance. (B) Local anesthetics were infiltrated along the way from subcutaneous to lateral SAP.

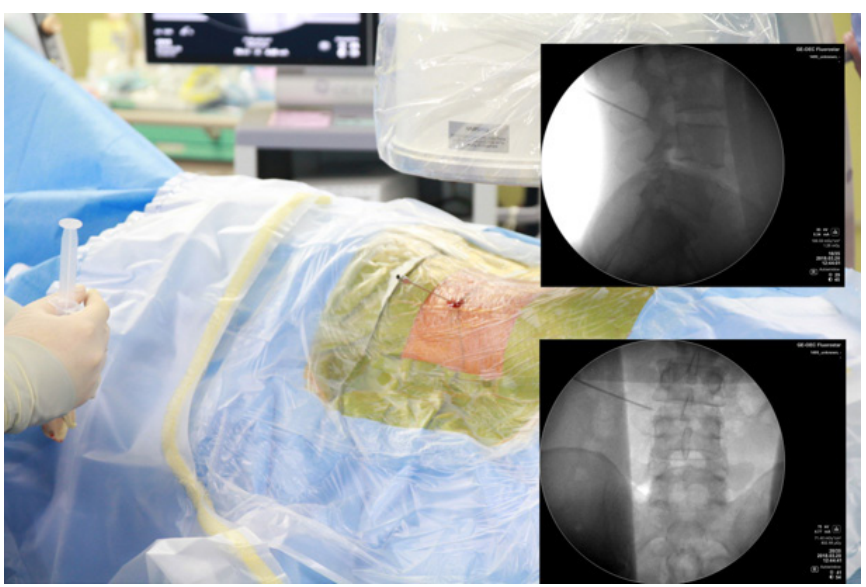

Figure 2. The intraoperative fluoroscopy showed cannulated needle landing on the lateral SAP.

al aspect of the SAP base.

\section{3) Endoscopic Foraminoplasty}

The endoscope of the working channel by $4.3 \mathrm{~mm}$ was used in operation. The radiofrequency coagulator (VANTAGE BIOTECH CO., LTD., Taoyuan, Taiwan) and grasping forceps are utilized to dissect soft tissue and then define the SAP under direct endoscopic visualization. The author used a high-speed diamond burr (VANTAGE BIOTECH CO., LTD., Taoyuan, Taiwan) to drill the ventral portion of the SAP base for widening the working space in the foramen. The foraminal ligament is then removed with forceps. Sometimes, a bulging disc in the foraminal area can be found and removed partially with forceps or shrank with a bipolar coagulator to maintain the endoscopic vision. After foraminoplasty, the working cannula can be inserted into the lateral recess of the spinal canal easily.

\section{4) Endoscopic Discectomy}

The migrated fragment was accessible and able to be pulled out from the canal. After partial discectomy, the annular defect was identified. Annuloplasty and epidural hemostasis are conducted with a bipolar probe. Adequate neural decompression is defined as the grossly free margin of the nerve roots with good pulsation.

\section{5) Monitoring During Operation}

The patient undergoing TELD was awake during the whole procedure. We didn't use the intraoperative neurophysiological monitor. The surgeon should communicate with the patient at each phase and be aware of the patient's response. If the patient reports leg dysesthesia during the procedure, the surgeon should halt and adjust the endoscopic or working instruments. Fluoroscopy is sometimes necessary to confirm the orientation of the surgical field. The operator can ask the patient to cough or wriggle the waist during operation to check if there is potentially herniated nucleus pulposus.

\section{RESULTS}

From May 2009 to June 2018, there were 126 patients undergoing TELD with foraminoplasty. The demographics are presented in Table 1 . The mean age of the patients was $50.7 \pm 17.4$ years (range from 14 to 82 years old). The mean follow-up time was 56.1 months (range from 17 to 130 months). At the one- 

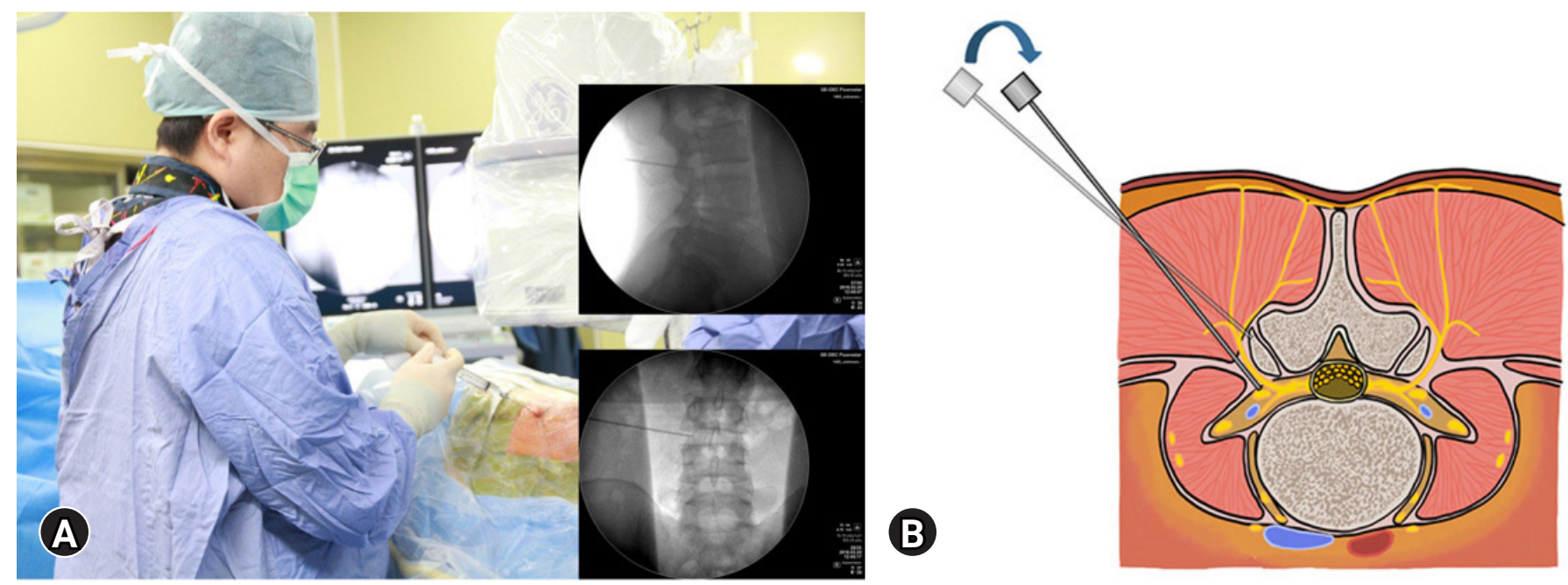

Figure 3. (A) The needle was then withdrawn slightly and inserted by a higher inclination angle into foramen. (B) The intraoperative fluoroscopy showed cannulated needle placed into foramen underneath the ventral surface of SAP.

Table 1. Patient characteristics

\begin{tabular}{lc}
\hline Characteristics & Values \\
\hline Gender & \\
$\quad$ Male & $93(71.4 \%)$ \\
$\quad$ Female & $33(28.6 \%)$ \\
Age (yr) & \\
Mean & $50.7 \pm 17.4$ \\
$<65$ & $90(71.4 \%)$ \\
$\geq 65$ & $36(28.6 \%)$ \\
Surgical level & \\
L2/3 & $5(4.0 \%)$ \\
L3/4 & $18(14.3 \%)$ \\
L4/5 & $94(74.6 \%)$ \\
L5/S1 & $8(6.3 \%)$ \\
L3/4 and L4/5 & $1(0.8 \%)$ \\
BMI & \\
$<30$ & $81(64.3 \%)$ \\
$\geq 30$ & $45(35.7 \%)$ \\
\hline
\end{tabular}

Values are $\mathrm{n}(\%)$ or mean \pm SD.

year follow-up, the VAS scores for leg pain significantly decreased from $7.4 \pm 1.7$ preoperatively to $0.3 \pm 0.9$ postoperatively $(\mathrm{p}<0.05)$. The ODI score significantly improved from $26.1 \pm 6.9$ to $2.8 \pm 4.0$ ( $\mathrm{p}<0.05)$ (Table 2). The operation time was within 2 hours in most cases (92\%). There was no conversion to open surgery. Minor adverse events included 13 patients reporting minor complications, including back soreness by two patients, ipsilateral leg numbness by eight patients, and significant wound pain. The minor complications were self-limited with conservative treatment. There was no neural injury, epidural hematoma, or iatrogenic instability requiring revision surgery
Table 2. Surgical outcomes

\begin{tabular}{lcc}
\hline & Values & p-value \\
\hline ODI & & $<0.001$ \\
Pre-op & $26.1 \pm 6.9$ & \\
Post-op & $2.8 \pm 4.0$ & \\
VAS & & $<0.001$ \\
Pre-op & $7.4 \pm 1.7$ & \\
Post-op & $0.3 \pm 0.9$ & NA \\
Operative time (hr) & & \\
$<1$ & $62(49.2 \%)$ & \\
$1-2$ & $54(42.9 \%)$ & NA \\
$>2$ & $10(7.9 \%)$ & \\
Complications & & \\
Soreness & $2(1.6 \%)$ & \\
Numbness & $8(6.3 \%)$ & \\
Severe wound pain (VAS $>4)$ & $3(2.3 \%)$ & \\
Recurrence & $7(6.3 \%)$ & \\
\hline
\end{tabular}

VAS: Visual Analogue Scale, ODI: Oswestry Disability Index.

in the current series. Patient satisfaction to surgery was excellent in 106 patients (84.1\%) and good in 13 patients (10.3\%) (Figure 4). Seven patients had recurrence within six months postoperatively. Two patients underwent repeated TELD successfully, and the other five patients underwent fusion surgery.

\section{DISCUSSION}

The indication of endoscopic spine surgery has been expanded in recent years $[18,19]$. As for lumbar disc herniation, the TELD without foraminoplasty might be limited in patients with a migrated fragment [20]. According to the anatomy of 


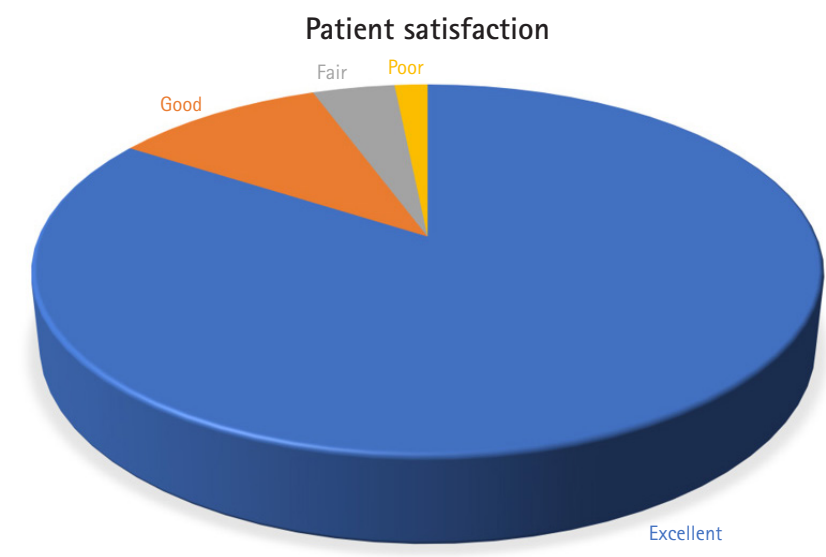

Figure 4. Patient satisfaction.

the intervertebral foramen, the SAP is the main obstacle in transforaminal endoscopic access to the epidural space in the spinal canal. Therefore, techniques of foraminoplasty were proposed to reach the migrated disc by widening the foramen during TELD. After the undercutting of the ventral part of the SAP, the safe working zone can be enlarged. So, the endoscopic trajectory can directly target the migrated fragment at the ventral epidural space [21]. This strategy can avoid exiting root irritation or injury due to increased cranio-caudal inclination for reaching migrated disc. The current study demonstrated the high success rate and safety in treating downward migration of lumbar disc herniation by TELD with foraminoplasty under local anesthesia. Patients could be satisfied with the painless endoscopic procedures, rapid recovery, and free from endotracheal intubation or urinary catheterization.

The first and most crucial step for any awake surgery is the techniques of anesthesia. The patient's cooperation is essential during the operation. Lumbar spine surgery could be one of the most painful procedures. Patients with lumbar disc herniation might be sensitive and irritable to painful sensations. Therefore, adequate analgesia is the core to make the patient cooperate during the surgery. Though TELD under epidural anesthesia is feasible, the intraoperative neurophysiological monitor is necessary to decrease the risk of neural injury [22]. Besides, it would not be cost-effective because of longer preparing time, higher cost of anesthesia and intraoperative monitor. Patients undergoing TELD might feel pain while skin incision, passing dilator through fascia, puncture into the annulus, and foraminoplasty with endoscopic burr or trephine. Therefore, local anesthesia with stepwise injection from the skin to the epidural space is enough to relieve pain during surgery. According to the current study, patients would be satisfied with the local anesthesia even undergoing complicated procedures like foramino- plasty. Besides, the patient may take less anesthetic risk when undergoing TELD, especially for the elderly or patients with multiple comorbidities. Post-anesthetic care is simple without endotracheal intubation or urinary catheterization. The perioperative and postoperative complications rates were lower with the protocol. The cost-effectiveness study also showed that TELD was more cost-effective than microdiscectomy by saving an additional net of $\$ 8,064$ per quality-adjusted life year [23].

There are some advantages to keeping patients awake during TELD. Iatrogenic neurological deficit is a critical issue of spinal operation. The rates of deficits after lumbar spine surgery ranged from $0.46 \%$ to $17 \%$ [24]. The utility of neurophysiological monitoring has shown the benefit of decreasing the risk of iatrogenic root injury $[25,26]$. While doing TELD under local anesthesia, patient's feedback during the operation can effectively replace the neurophysiological monitor. The diluted lidocaine can block the painful feeling but spare the tactile sensation during operation. Patients will report feeling like electric shock at the ipsilateral leg when the nerve root is irritated. The patient's feedback in real-time is straightforward without interpretation of neurophysiological electrography. The risk of injury to the neural structure is minimized and neurophysiological monitoring is unnecessary. Moreover, the patient can report the immediate improvement after decompression of the nerve root. When patients undergo TELD in lateral decubitus position, the surgeon can do a straight leg raising test during operation to confirm the effectiveness.

The extent of discectomy is a critical issue for the recurrence of the lumbar disc. The previous studies revealed that limited discectomy was related to a higher risk of recurrence than subtotal discectomy $[27,28]$. However, an aggressive discectomy may cause more postoperative back pain $[27,29]$. There is no standard to determine the adequate extent of discectomy during the operation. The authors proposed an easy way to determine the endpoint during the awake operation. While asking the patient to cough or wriggle the waist, the surgeon can identify the loose disc fragment during operation. It is difficult to do similar tests when patient is under general anesthesia. Therefore, discectomy during awake surgery might balance the disc preservation and recurrent risk during lumbar discectomy.

There are limitations in the current report. It is a retrospective study, and there is a lack of comparative group and prospective data to evaluate differences between anesthetic techniques. Though this technique might be suitable for all TELD, the foraminoplasty is an advanced technique, and the learning curve remains steep for beginners to achieve favorable outcomes steadily. Different surgical techniques and technologies 
may affect the outcomes and patient satisfaction. The current study emphasized short-term results regarding the anesthetic technique but omitted the discussion of long-term outcomes.

\section{CONCLUSION}

Ambulatory surgery is the trend of minimally invasive spine surgery. With skilled anesthesia in awake surgery, the surgeon can widen the intervertebral foramen during transforaminal endoscopic spine surgery to reach the migrated fragment. Intraoperative feedback from patients during awake surgery assures safety by avoiding neural injury. Though TELD has advanced and varied with the expanding indications, the technique of foraminoplasty remains the primary and core skill in the developing field.

\section{CONFLICT OF INTEREST}

No potential conflict of interest relevant to this article.

\section{ACKNOWLEDGEMENTS}

The authors thank the project assistant, Ms. Ying-Chieh Chen, for helping the program.

\section{REFERENCES}

1. Kambin P. Arthroscopic microdiskectomy. Mt Sinai J Med 1991;58:159-164.

2. Kambin P, O'Brien E, Zhou L, Schaffer JL. Arthroscopic microdiscectomy and selective fragmentectomy. Clin Orthop Relat Res 1998;(347):150-167.

3. Mathews HH. Transforaminal endoscopic microdiscectomy. Neurosurg Clin N Am 1996;7:59-63.

4. Savitz MH. Same-day microsurgical arthroscopic lateral-approach laser-assisted (SMALL) fluoroscopic discectomy. J Neurosurg 1994;80:1039-1045.

5. Mayer HM, Brock M. Percutaneous endoscopic discectomy. surgical technique and preliminary results compared to microsurgical discectomy. J Neurosurg 1993;78:216-225.

6. Lewandrowski KU, Dowling Á, de Carvalho PST, Calderaro AL, Dos Santos TS, de Lima E Silva MS, et al. Indication and contraindication of endoscopic transforaminal lumbar decompression. World Neurosurg 2021;145:631-642.

7. Akçakaya MO, Yörükoğlu AG, Aydoseli A, Aras Y, Sabancı PA, Altunrende ME, et al; Serum creatine phosphokinase levels as an indicator of muscle injury following lumbar disc sur- gery. Comparison of fully endoscopic discectomy and microdiscectomy. Clin Neurol Neurosurg 2016;145:74-78.

8. Ahn SS, Kim SH, Kim DW, Lee BH. Comparison of outcomes of percutaneous endoscopic lumbar discectomy, open lumbar microdiscectomy for young adults. a retrospective matched cohort study. World Neurosurg 2016;86:250-258.

9. Barber SM, Nakhla J, Konakondla S, Fridley JS, Oyelese AA, Gokaslan ZL, et al. Outcomes of endoscopic discectomy compared with open microdiscectomy, tubular microdiscectomy for lumbar disc herniations. a meta-analysis. J Neurosurg Spine 2019;31:802-815.

10. Kim M, Lee S, Kim HS, Park S, Shim SY, Lim DJ. A comparison of percutaneous endoscopic lumbar discectomy, open lumbar microdiscectomy for lumbar disc herniation in the Korean. a meta-analysis. Biomed Res Int 2018;2018:9073460.

11. Liu X, Yuan S, Tian Y, Wang L, Gong L, Zheng Y, et al. Comparison of percutaneous endoscopic transforaminal discectomy, microendoscopic discectomy, microdiscectomy for symptomatic lumbar disc herniation. minimum 2-year follow-up results. J Neurosurg Spine 2018;28:317-325.

12. Chen KT, Wei ST, Tseng C, Ou SW, Sun LW, Chen CM. Transforaminal endoscopic lumbar discectomy for L5-S1 disc herniation with high iliac crest. technical note and preliminary series. Neurospine 2020;17:S81-S87.

13. Hasan S, Härtl R, Hofstetter CP. The benefit zone of full-endoscopic spine surgery. J Spine Surg 2019;5:S41-S56.

14. Choi G, Lee SH, Lokhande P, Kong BJ, Shim CS, Jung B, et al. Percutaneous endoscopic approach for highly migrated intracanal disc herniations by foraminoplastic technique using rigid working channel endoscope. Spine (Phila Pa 1976) 2008;33:E508-E515.

15. Yeung AT, Yeung CA. Advances in endoscopic disc and spine surgery: foraminal approach. Surg Technol Int 2003;11:255263.

16. Zhang L, Yang J, Hai Y, Yin P, Ding Y, Xu C, et al. Relationship of the exiting nerve root and superior articular process in Kambin's triangle: assessment of lumbar anatomy using cadavers and computed tomography imaging. World Neurosurg 2020;137:e336-e342.

17. Schubert M, Hoogland T. Endoscopic transforaminal nucleotomy with foraminoplasty for lumbar disk herniation. Oper Orthop Traumatol 2005;17:641-661.

18. Chen KT, Jabri H, Lokanath YK, Song MS, Kim JS. The evolution of interlaminar endoscopic spine surgery. J Spine Surg 2020;6:502-512.

19. Khandge AV, Sharma SB, Kim JS. The evolution of transforaminal endoscopic spine surgery. World Neurosurg 


\section{1;145:643-656.}

20. Choi KC, Lee DC, Shim HK, Shin SH, Park CK. A strategy of percutaneous endoscopic lumbar discectomy for migrated disc herniation. World Neurosurg 2017;99:259-266.

21. Sairyo K, Higashino K, Yamashita K, Hayashi F, Wada K, Sakai $\mathrm{T}$, et al. A new concept of transforaminal ventral facetectomy including simultaneous decompression of foraminal and lateral recess stenosis: technical considerations in a fresh cadaver model and a literature review. J Med Invest 2017;64:1-6.

22. Fang G, Ding Z, Song Z. Comparison of the effects of epidural anesthesia and local anesthesia in lumbar transforaminal endoscopic surgery. Pain Physician 2016;19:E1001-E1004.

23. Choi KC, Shim HK, Kim JS, Cha KH, Lee DC, Kim ER, et al. Cost-effectiveness of microdiscectomy versus endoscopic discectomy for lumbar disc herniation. Spine J 2019;19:11621169.

24. Ghobrial GM, Williams KA Jr, Arnold P, Fehlings M, Harrop JS. Iatrogenic neurologic deficit after lumbar spine surgery: a review. Clin Neurol Neurosurg 2015;139:76-80.

25. Bosnjak R, Makovec M. Neurophysiological monitoring of S1 root function during microsurgical posterior discectomy using H-reflex and spinal nerve root potentials. Spine (Phila Pa 1976) 2010;35:423-429.

26. Macdonald DB, Stigsby B, Al Homoud I, Abalkhail T, Mokeem A. Utility of motor evoked potentials for intraoperative nerve root monitoring. J Clin Neurophysiol 2012;29:118-125.

27. Carragee EJ, Spinnickie AO, Alamin TF, Paragioudakis S. A prospective controlled study of limited versus subtotal posterior discectomy: short-term outcomes in patients with herniated lumbar intervertebral discs and large posterior anular defect. Spine (Phila Pa 1976) 2006;31:653-657.

28. Wera GD, Dean CL, Ahn UM, Marcus RE, Cassinelli EH, Bohlman HH, et al. Reherniation, failure after lumbar discectomy. a comparison of fragment excision alone versus subtotal discectomy. J Spinal Disord Tech 2008;21:316-319.

29. McGirt MJ, Ambrossi GL, Datoo G, Sciubba DM, Witham TF, Wolinsky JP, et al. Recurrent disc herniation and long-term back pain after primary lumbar discectomy: review of outcomes reported for limited versus aggressive disc removal. Neurosurgery 2009;64:338-344; discussion 344. 